\title{
AI Agents, Humans and Untangling the Marketing of Artificial Intelligence in Learning Environments
}

\author{
Isabel Pedersen \\ Faculty of Social Science and \\ Humanities \\ Ontario Tech University \\ Oshawa, Canada \\ Isabel.pedersen@ontariotechu.ca
}

\author{
Ann Hill Duin \\ Writing Studies Department, \\ College of Liberal Arts \\ University of Minnesota \\ Minneapolis, USA \\ ahduin@umn.edu
}

\begin{abstract}
This exploratory study identifies the tangling of proposed relationships between human and nonhuman agents by providing an analysis on how AI technologies are marketed for learning subjects through a critical discourse analysis of corporate advertisements. We ask: Amid these emerging technologies, how are humans and AI technologies framed as agents with agency? How are learners framed by corporate advertising as part of this blurring? We used a public, open-access cultural analytics database and repository, Fabric of Digital Life ('Fabric', https://fabricofdigitallife.com/), to identify a set of artifacts as a dataset for such analysis. Results indicate that advertising promotes corporate products while also promoting idealized social practices for human-computer interaction and humanrobot interaction in learning contexts. Using AI to automate relationships between students and teachers frames AI systems as authorities in both robot and non-robot platforms, blurring and minimizing student and instructor agency in learning environments.
\end{abstract}

\section{Introduction}

Although much has been written about empowering learners in networked learning environments, little has been written about how emerging educational AI technologies are marketed to consumers in the name of learning. This paper contends that representations of agency, rather than serving to ground interactions amongst learners, teachers, and non-human AI agents in learning environments, obfuscate proposed relationships. For example, on April 18, 2016, Anita Schjøll Brede, CEO
Iris.AI explains the broad intent for her new company's use of AI technology:

The abundance of knowledge that we have today is unprecedented, but our human minds do not have sufficient computational power to process it all, or easier said, we can't read and understand everything. Every single day, three thousand papers are published within science, technology and medicine alone. Three thousand. Not to mention the millions that are already out there. So a lot of these resources end up in the digital equivalent of a dusty old drawer... But what if we had a brain, a really big, really powerful brain that could read and make sense of all of this. Well, that is what we are building with Iris AI [1].

In this TEDx talk, Brede points to a gap in consumption, that research is not being read despite the Internet's capacity to publish information and circulate it. She also explains the gap in terms of human capacities, a lapse in learning, that "we can't read and understand everything" [1]. However, the gap will be remedied by AI and specifically by Iris, the AI agent created by her company. Later in the talk, she highlights the transformation from assistant to personified researcher, "Iris just might be able to become a researcher herself" [1]. Applying a critical lens, Crawford identifies such AI anthropomorphization as contributing to a larger corporate mythology surrounding nonhuman systems. She explains, "This perspective assumes that with sufficient training, or enough resources, humanlike intelligence can be created from scratch, without addressing the fundamental ways in which humans are embodied, relational, and set within wider ecologies" [2: 13]. Agent narratives, like that of Iris.AI, proposed and marketed during the development, emergence, and 
adoption of AI technology, cast expectations on future learners, teachers, and instructional designers.

This paper addresses the tangling of proposed relationships between human and non-human agents by providing an analysis on how AI technologies are marketed for learning subjects through a critical discourse analysis of corporate advertising. We assess agency and its placement in these texts to determine how students (and other demographics of learners) are invited to engage with AI agents including virtual assistants, robots, and implicit AI agents.

We do not mean to imply that entangled human and non-human collaboration in learning is an undesirable state. Rather, we seek to untangle marketing goals imposed upon the public in the context of complex learning environments. We acknowledge the heritage of writers that characterize entanglement as a characteristic of post-digital human and non-human collaboration [3] [4] [5]. For example, Hayles writes:

While traditional ethical inquiries focus on the individual human considered as a subject possessing free will, such perspectives are inadequate to deal with technical devices that operate autonomously, as well as with complex human-technical assemblages in which cognition and decision-making powers are distributed throughout the system. I call the latter cognitive assemblages [3: 4].

Hayles' term, cognitive assemblages, provides a means to interpret distributed decision making between humans and AI agents. Likewise, Siemens writes, "Our learning peers are not exclusively human; they are also algorithms and automated agents" [6].

We observe that boundaries between human and artificial decision-making are blurred because learning takes place across distributed multiple platforms at school and at home (e.g. Home-based Alexa helps students with homework). Therefore, we address a key research question in the Call for Proposals: "Can we effectively develop agency, trust, and interventions to support learning in complex environments where boundaries between human and artificial decisionmaking become blurred?" And more specifically, How are both humans and AI technologies framed as agents with agency? How are learners framed by corporate advertising as part of this blurring?

We make the argument that as corporations market AI technologies for learning contexts (k-12 students, postsecondary students and/or lifelong learners and professionals), human abilities and capacity to learn are framed in a machine-centric way, that is, as being in need of more efficient and productive processes aided by these technologies as a means to keep up which in turn results in greater learning. A subtext result is confusion over the term learning as related to Machine-Learning which contextualizes human learners in a subordinate role. As Shneiderman notes, the disturbing assumption about automation is that "increases in automation must come at the cost of lowering human control" [7: 495]. This reduction and oversimplification entangles our understanding of human-machine decision making; moreover, it obfuscates the ability to foreground positive learning outcomes made possible through the use of AI in learning environments.

\section{Literature review}

People are increasingly adept at using AI agents including Siri, Alexa, and Google assistant. Each assistant's AI agency, defined as the capacity to act autonomously (act independently), to adapt (react and learn from changes in the environment) and to interact (to perceive and respond to other human and artificial agents) [8], makes these useful. In a previous publication, we explain how the popularity of virtual assistants has helped spawn the creation of 'virtual humans' that are lifelike, virtual personas with nuanced facial, gestural, and spoken interaction. These screen-based, virtual humans mimic human physical reactions to be made to appear empathetic, unique, and mildly emotional [9]. Moreover, digital publishing platform companies increasingly require human writers to employ AI for automating and analyzing writing tasks across a range of functions that are becoming ever more tangled [9]. Students can drop rough ideas into a writing program and "the software will recommend language to express what he or she is trying to say - cocreating with the human based on his or her ideas" [10]. These AI tools are designed to assume agentive roles of editorship and authorship; e.g., AI writer, Wordsmith (Automated Insights), Inferkit, and United Robots all promote automated writing. AI-Writer describes itself as "a service that helps you create better content in less time! Just feed our algorithm a headline and it will do all the research work for you. Yes, it's really that simple!"

Robots are now deployed as teachers, tutors, peers, tools and caregivers, and speculation surrounding the combination of autonomous technologies and humanoid or social robots is rampant. Scholarship and debate on robot friendship and empathetic AI suggests that development in this sector will increase $[11,12$, 13]. As a result, Hayles writes that the dynamic relationship between humans and nonhumans will undergo profound change: "Each technical object has a set of design specifications determining how it will behave. When objects join in networks and interact/intraact with human partners, the potential for 
surprises and unexpected results increases exponentially" [3: 84]. De Visser et al. also emphasize that such "autonomy will surprise human partners to an even greater extent than simple automated systems" [14].

These AI applications and adaptive learning technologies related to teaching and learning are expected to grow significantly. According to the 2021 AI Index, "technical advances have started to outpace the benchmarks to test for them" [15]. The most recent 2021 U.S. Horizon Report notes that "AI has advanced to 'self-supervised learning,' an advance on deep learning in which a computer system can learn from raw or non-labeled data." This report stresses the opportunity "to thoroughly rethink the curriculum and associated academic programs in a way that will better serve "Generation AI" [16]. In terms of how universities are responding to this growth, we share one example: the Technical University of Eindhoven in the Netherlands announced the launching of an Artificial Intelligence Systems Institute with 50 new professorships for education and research in AI [17]. The U.S. National Science has also established 11 new National Artificial Intelligence Research Institutes in partnership with universities to accelerate innovation in many sectors including education [18].

In their systematic review of 2656 studies between 2007 and 2018 on AI applications higher education, Zawacki-Richter et al. synthesized 146 articles, determining four AI application areas: profiling and prediction, assessment and evaluation, adaptive systems and personalization, and intelligent tutoring systems. Amid rapidly expanding applications, they note the lack of critical reflection on the challenges and risks, the weak connection to pedagogy, and the need for ethical and educational approaches in AI applications [19]. In a similar study, Guan, Mou, and Jiang reviewed 400 studies over the past 20 years on the application of AI and deep learning approaches in teaching and learning, identifying major research themes and trends that indicate "the decline in conventional tech-enabled instructional design research and the flourishing of student profiling models and learning analytics" [20]. These studies provide an overview of how AI has been conceptualized in education; they include detail on AI computer-assisted instruction, virtual and augmented reality in education, predictive modeling and learning analytics. While authors in these studies mention that "companies" are proceeding in their expanding development of AI agents and the integration of AI in learning environments, no studies appear to identify how learners are framed by corporate marketing amid such development, nor how humans and AI technologies are framed as agents with agency.
Most recently, Cox explores the impact of AI and robots on higher education. Cox emphasizes that recent AI and robotic developments in higher education are surrounded by controversy in terms of what is technically possible versus what is practical, pedagogical, and desirable. We agree with Cox and others who note that AI and robotics will change how education works, what learning is like, the role of instructors and researchers, and how our institutions work. Cox uses design fictions to imagine future scenarios of AI and robotics use: "Design fictions create a speculative space in which to raise questions about whether a particular technology is desirable, the socio-cultural assumptions built into technologies, the potential for different technologies to make different worlds, our relation to technology in general, and indeed our role in making the future happen" [21]. Cox's design fictions build on technologies and genres currently present in support of human and non-human agent relationships: intelligent tutoring systems through use of sensors for adaptability, text and data mining, automated writing as noted earlier, and conversational agents. We appreciate Cox's fictional renditions of each technology's envisioned use as a means to open up "dimensions of debate around AI" as they present depictions of "social, ethical, pedagogic and management issues of automation through AI and robots on HE [higher education]" [21]. Again, little analysis appears to address how learners are framed by corporate advertising amid such design fictions and subsequent development.

Likewise, studies of learning analytics applications illustrate the vast algorithmic and analytical capabilities made possible with technology that are now woven into the seemingly objective, structural properties of our institutions. Duin and Tham in their 2020 case study of the adoption of the Canvas learning management system (LMS) at two universities, stress the importance of instructors becoming more familiar with levels of access to academic and learning analytics, more acquainted with the analytical capabilities in LMSs, and more mindful of implications of learning analytics stemming from LMS use in this case of writing pedagogy [22]. Their study makes visible the serious lack of student involvement in and access to learning analytics and how their profiles are used/viewed by instructors and administration. In this case, the application's agency, designed by companies, inherently has a corporate frame as its main client. Duin and Tham argue that researchers and instructors should collaborate with instructional designers and analytics specialists, attain data and analytics literacy, use available data ethically, and create sustainable frameworks for programmatic development. 
It remains unclear as to the agency that students have in the above monitoring scenarios as well as in student use of the myriad of learning management systems. In the 2021 collection by Dohn et al., Mobility, Data, and Learner Agency in Networked Learning, contributors address additional issues of agency: cognitive load in these learning environments; student empowerment when given the opportunity to become co-researchers of semantic data; the power of agency that's possible in student communities of practice; and how boundary objects surrounding transfer, translation, and transformation work to increase student agency for traversing the various approaches to learning [23]. Throughout all the above investigations, while attention is paid to understanding the student learner, again, less attention is directed toward interpreting the client, the user, the learner, and how each AI application is marketed as a result of this interpretation.

\section{Theory}

Key to this study is envisioning the design of automated systems without foregoing the role of human decision-making and learner agency. Hayles describes cognitive assemblages within value-based terms:

We need to recognize that when we design, implement, and extend technical cognitive systems, we are partially designing ourselves as well as affecting the planetary cognitive ecology: we must take care accordingly. More accurate and encompassing views of how our cognitions enmesh with technical systems and those of other life-forms will enable better designs, humbler perceptions of human roles in cognitive assemblages, and more life-affirming practices as we move toward a future in which we collectively decide to what extent technical autonomy should and will become increasingly intrinsic to human complex systems [3: 141]

To emphasize one point, Hayles expresses the need to discover "how our cognitions enmesh with technical systems" before we propose technologies for learners; ultimately, "we are partially designing ourselves" in partnerships with autonomous systems [3: 141].

To add dimension to understanding the integration of AI into learning environments, we pursue a qualitative critical approach to interpret the ways in which corporate discourses construct the role of learning subjects in relationships with these emergent AI technologies. Critical discourse analysis generally assumes "that institutions act as gatekeepers to discursive resources; [leading to] power and resource imbalances between 'speakers' and 'listeners" [24].
Critical discourse analysis "is particularly interested in linguistic manifestations of power (Wodak \& Meyer, 2009). The exercise of power influences knowledge, beliefs, understandings, ideologies, norms, attitudes, values, and plans, and CDA [Critical Discourse Analysis] seeks to uncover, reveal, and disclose implicit or hidden power relations in discourse (Van Dijk, 1993)" [24: 119]. In the tradition of Norman Fairclough and Teun A. van Dijk, Mullet writes specifically of educational research, stating that "the analyst's goal is to bring about change through critical understanding." In line with this goal, we identify key themes related to obfuscation of agency involving learning subjects in a specific discourse, marketing related to AI technologies geared to emergent educational media. We seek to "disclose implicit" power relations already implied in corporate discourses of pre-release technologies that not only introduce a product, but also introduce or habituate learners to practices involving AI agents.

\section{Methodology}

We used a public, open-access cultural analytics database and repository, Fabric of Digital Life ('Fabric', https://fabricofdigitallife.com/), to identify a set of artifacts as a dataset for such analysis. Growing since 2013, Fabric holds more than 4400 artifacts. The thematic focus for this repository consists of platforms of embodied human-computer interaction, namely, carryables, wearables, implantables, ingestibles, embeddables, robotics, and ambient platforms. Fabric's content grows through the work of a community of researchers from Ontario Tech University, University of Minnesota, Temple University, Texas Tech University, and Canada Science and Technology Museum. It tracks the emergence of embodied computing technologies by providing extensive classification metadata. Researchers contribute to the database through new content artifacts and the metadata associated with each for building thematic research collections.

Curated by both established and student researchers, dozens of collections work to situate emergent, embodied, technologies within broader digital cultural discourses. Fabric's aim is to contextualize technology emergence within both traditional and non-traditional media genres such as magazine journalism, broadcast news, marketing outlets, tradeshow videos, video games, government publications, films, and academic research venues to reveal how digital technology is evolving. For example, an invention might be announced in an academic journal article, celebrated in a popular science magazine, and depicted as a fictional artifact 
in a video game. All of these instantiations of an invention contribute to its emergence. In addition to recognizing multiple overlapping discourses, Fabric has developed a keyword classification system that recognizes a human-centred approach to technology emergence through several keyword categories. We next share the specific methodology for use of the Fabric repository for investigation of human and nonhuman relationships as we work to untangle the integration of artificial intelligence in learning environments.

To explore the above research questions, we followed Mullet's general critical discourse analytic framework to bring a qualitative critical approach to interpret the ways in which discourses construct the role of learning subjects in relationships with emergent AI technologies. We followed the stages of analysis including, select the discourse, locate data sources, explore the background of each text, identify overarching themes, analyze external and internal relations in the texts, and interpret the data [24: 122]. To select and locate data sources, we identified relevant advertisements from the Fabric repository using broad keywords searches, analyzed the resulting sets, and continued filtering sets of artifacts down to generate a list of specific artifacts.

First, we found that 385 artifacts of Fabric's entire collection of 4454 artifacts were tagged with the Augment keyword learning, which indicates that a technology is involved in some form of human learning, either in a learning environment or any context (e.g. non-students learning another language using a wearable device, elderly persons learning a fitness activity, individuals using a self-improvement app to learn about their moods and behaviors or professionals learning to write better for work). Second, we continued to filter the artifacts according to technology keywords; 160 of the 385 learning artifacts were tagged with the technology keyword Artificial Intelligence (AI). AI technologies include numerous subcategories such as machine learning, neural networks, or deep learning, however, all of these are included under the broader keyword, Artificial Intelligence (AI). At this point, the set of 160 artifacts still covered a disparate range of learningbased themes involving AI and mobile or wearable devices (e.g. sports, social interaction, aging, arts, work, etc.). The keyword Education allowed us to better orient the set to learning environment domains; this filter brought the set to 51 artifacts published from 2014-2021. All of these artifacts had an association with Education as an industry sector. Finally, from the set of 51, specifically identified corporate advertising videos to reveal more detail on the proposed relationships between human and non-human agents made by companies through marketing claims.

We discovered that some artifacts frame robots as learners and humans as teachers, pointing to machine centricity. We removed several artifacts from our set based on this reasoning. For example, Kiki robot's promotional advertising (https://www.kiki.ai) is nearly entirely pitched as a learning robot framed as consumers teaching it as part of an entertaining practice.

The resulting dataset, 17 video advertisements for newly emerged or emerging AI technologies, provided a means to analyze inventions promoted as having an educational function. These included advertising for consumer products/solutions on the market, corporate artifacts for companies that are no longer active (discontinued products or companies), and products that are still in pre-release research and development but are advertised as a future product. For each video, we also looked at materials at the companies' websites to determine that each targeted learning environments or implied them as one of their intended domains. The set of 17 can be further analyzed under specific hardware platforms: seven are carryable/mobile technologies that are used on phones, tablets or laptops; seven are humanoid robots; and three are wearables designed to be worn on the body. (See Appendix 1 for the final set of 17, a listing of the complete set of artifacts and relevant organizations.)

Not included in this set are purely academic or research-based artifacts, which are relevant to the discourse of AI agency and education but are not yet contextualized as commercial entities. For example, Jill Watson (inspired and supported by IBM Watson) is a virtual teaching assistant for answering questions based on educational texts. It has been developed by the emPrize team of faculty, staff and students associated with Georgia Institute of Technology's Design \& Intelligence Laboratory. The team is led by Dr. Ashok Goel who has released a TEDx promoting the idea of AI teaching assistants. The Jill Watson project has been used in learning environments, including actual university courses.

\section{Results}

Results indicate that marketing not only promotes corporate products, it promotes idealized social practices for human-computer interaction and humanrobot interaction in learning contexts. In keeping with the critical discourse analysis agenda, we sought to make explicit the concealed power relationships reproduced in these texts in light of the research questions. We identified three themes from this dataset to classify blurred boundaries depicted in the ads. At 
the same time, we acknowledge that in this marketing genre, design fictions are deliberately embedded in promotional materials for AI systems.

\subsection{Blurred target audiences}

First, devices are promoted for multiple markets and audiences, rather than concentrating primarily on learners embedded in a learning environment. While the set of artifacts has been classified as applicable to educational domains, many artifacts point to multiple intended audiences and consumers, leading to blurred portraits of agency in these relationships. For example, the Misty robot (see Figure 1) is marketed as an office robot, a home companion robot for aging persons, a library robot, a therapy robot, and an educational support robot. Interdiscursivity in the marketing mars the opportunity to properly assess how AI agents will empower human learners.

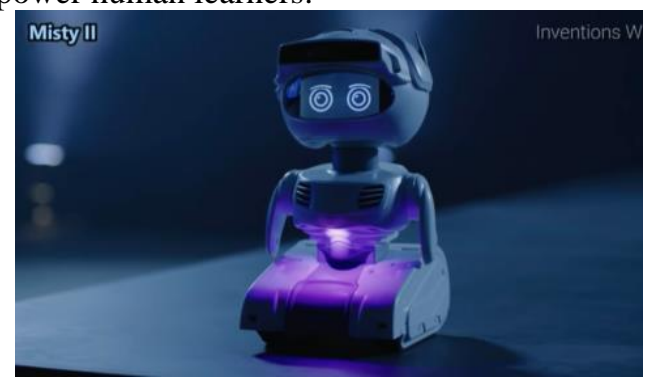

Figure 1. Screen capture from Misty II Is Here video

\subsection{AI assistantship and learning}

Second, human and non-human relationships are depicted as assistantship. Five of the 17 artifacts are classified as Virtual Assistants for mobile/carryable devices. Several are applications pitched as providing collaborative writing assistance (see figure 2). We note that these artifacts use more transparent, less blurred language about collaborating with AI assistants for writing technologies. This result aligns strongly with our previous study of future collaborative writing environments [9].

Some products are proposed as anthropomorphized AI agents framed as superior to human learners. Phrases in the advertising position humans as needing the technology in order to navigate a changing world. Pointing to vast quantities of research information made available on the Internet, for example, a participant in the iris.ai video states "It is really difficult for people to keep up with this knowledge" (Iris.AI). Fove Inc.'s website states of its virtual reality product "Unleash Human ability, realizing a world of virtually limitless possibility. . . with the aid of XR".

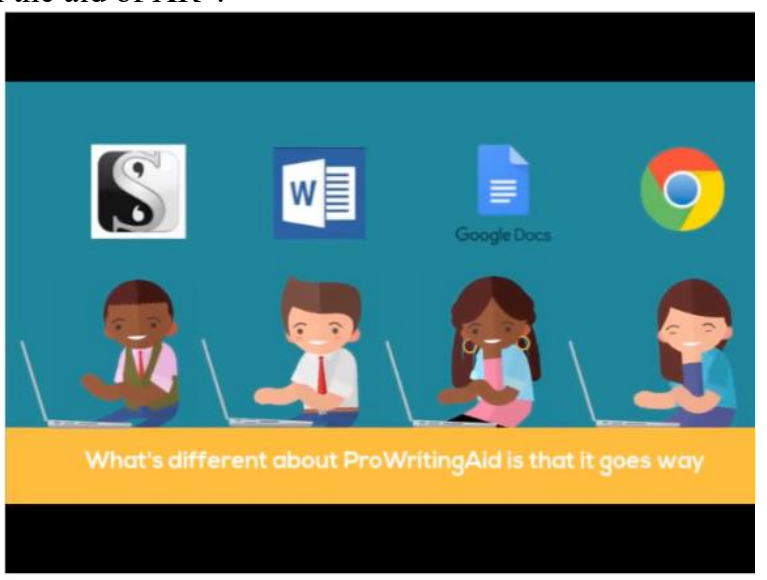

Figure 2: Screen capture from ProWritingAid: Your Personal Writing Coach video

\subsection{Robots as teachers in learning contexts}

Third, several artifacts depict robots as teachers. Appearing in often subtle ways, there is a pronounced trend toward automation of traditional teacher's roles. Seven of the 17 artifacts involve humanoid robots: Misty, Sophia, Little Sophia, Canbot, Zenbo, Miko, and Jibo. Five of the seven are tagged with the keyword, children. Child learners are visually depicted with a robot helping them with homework or answering factual questions (see Figure 3). Robots appear in learning spaces and teaching occurs in classrooms as well as in homes (e.g., bedrooms, living rooms). Both Sophia and Little Sophia reference the theme of helping girls learn STEM subjects. The Zenbo robot acts as a medical assistant and a teacher in an early childhood classroom, helping to take children's temperatures, alongside a human teacher. However, learners often are depicted with only the Robot teacher present.

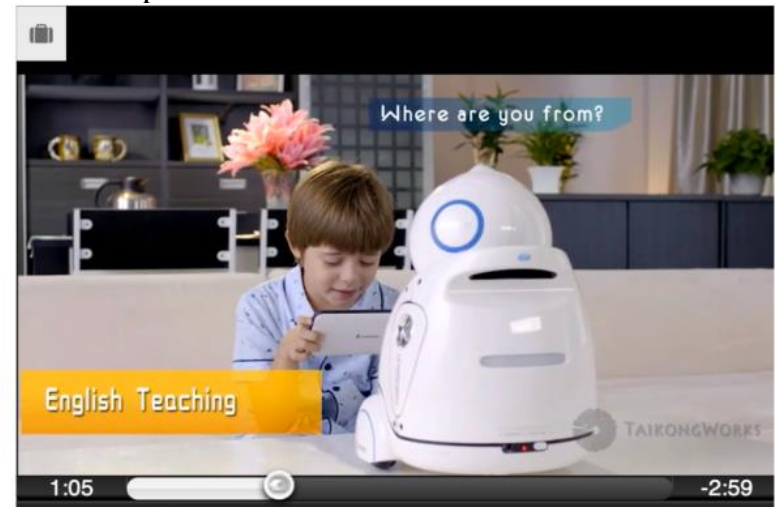

Figure 3. Canbot's robot teaches a child at home without the presence of a human teacher 


\section{Discussion}

This study queries the concept of agency in complex learning environments that are increasingly adopting new AI technologies by exploring two questions: How are both humans and AI technologies framed as agents with agency? How are learners framed by corporate advertising as part of this blurring? This study begins to explore the tangling of proposed relationships between human and nonhuman agents by providing an analysis on how AI technologies are marketed for learning subjects through a critical discourse analysis of corporate advertising. Results indicate that corporate marketing blurs target audiences, adding learning environments to a myriad of uses. Learners are urged to carry and engage with virtual assistants as perhaps the one means to keep up with mounting information, and the definition of teacher is broadened through robots positioned to contextualize humans in a subordinate role.

Often meant to identify overt power relationships and inequity, a critical discourse analysis approach enabled us to identify subtle markers of obfuscation in these depictions. We find that using AI to automate relationships between students and teachers frames AI systems as authorities in both robot and non-robot platforms, minimizing the depiction of learner agency in these representations. This result most requires further research and ethical consideration for learning environments. For example, one of the companies, Find Solution AI, uses algorithms to determine students' emotional state to enable teachers to monitor student concentration and motivation through emotion recognition (see Figure 4). Similar to the work of Kim, the intent is to drive students to be more motivated by tracking their facial micro-gestures and having teachers react according to the AI's result [25]. Flagged in mainstream media, the goal is to automate learners' intent, "to make humans less inscrutable and easier to predict at scale" [26]. However, the validity of monitoring facial movements for this purpose, judging emotional states, has been questioned and deemed as lacking validity [27]. Again, teacher agency becomes blurred because AI agency is so readily assumed to be authoritative over the learner's behaviours.

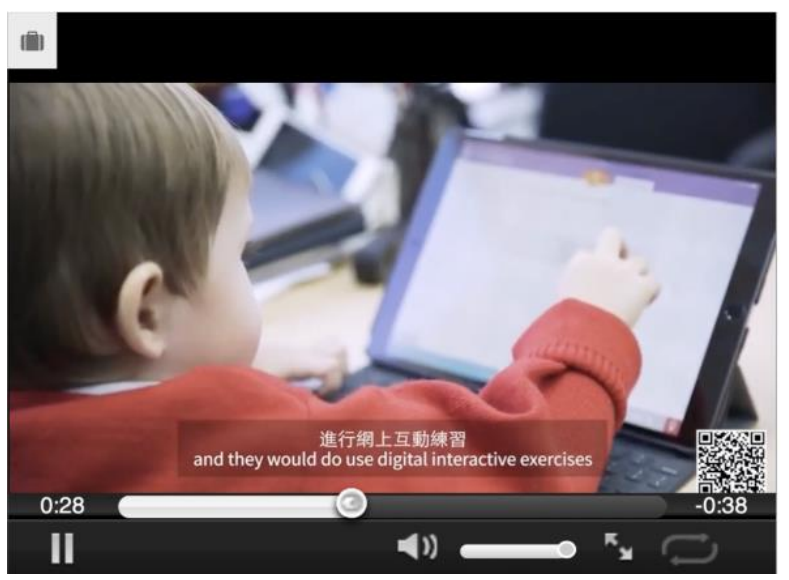

Figure 4: Screen capture from Solution AI big data

Papacharissi, professor and prolific author of books on the social consequences of technology, presciently writes that "we imagine and then design machines to mimic and replace us" [28]. As humanoid robots become more sophisticated, developers and marketers will continue to frame these in multiple agentive roles for purposes of profit. The first discursive theme refers to the way that texts assume multiple intended audiences and consumers, leading to blurred portraits of agency. Pepper robot made by Softbank is now being used deliberately as both entertainer and teacher: "Pepper's conversational skills and ability to provide an edutainment [role] both in terms of education or cognitive training (quizzes and games) and in terms of increasing the users' confidence" [29]. Again, Hayles writes that "because humans and technical systems in a cognitive assemblage are interconnected, the cognitive decisions of each affect the others, with interactions occurring across the full range of human cognition" [3: 118]. To drive ethical design for AI agents in learning environments, we argue that learners in learning contexts need to be included as partners in decision-making systems with recognition of their unique goals, affordances, and potential vulnerabilities. The conflation of robots for therapy, teaching, or officework, for example, minimizes the potential for agency in each of these scenarios. Ryberg and Sinclair speak to this ongoing socio-material turn that is taking place, suggesting that we should understand entities such as avatars (and we add humanoid robots here) as socio-material entities [30]. In short, we can no longer view these entities as disconnected from our material world.

Techno-utopianism is clearly common across the dataset of artifacts, bolstering each of the above themes with urgency and energy. For example, in one video, Andrew Ochao, founder of Waverly Labs, shares, "Imagine being able to snap your fingers and become fluent in 20 languages." Use of the Fabric 
archive provides a means to contextualize each artifact within a discourse of emerging technologies as social phenomena rather than as purely technical, commercial or heavily-hyped entities. Language that promotes AI systems instrumentalizes human intent, activities, and goals, while AI agency hinges upon degrees of successful automation. Fabric metadata provides a means to analyze keywords related to human agents in traditional learning environments and to pinpoint ways that humans and non-humans interact.

\section{Conclusion}

This study explored the relationship between human and non-human agents, analysing how AI technologies are marketed for learning subjects through a critical analysis of corporate advertising. This exploratory research provides an initial methodology for identifying specific Fabric artifacts and analyzing them for a specific research question. Using this methodology led to the identification of three key themes. By obfuscating agency in marketing, these video artifacts promote understanding of the blurred role for teachers and learners adopting and adapting to AI technologies.

A next step for this reported research is to foster continued development of critical literacies for teachers and students to engage as critical, knowledgeable consumers of emerging technologies. Such knowledge positions us to untangle the integration of AI in learning environments. As Dohn et al. write, "There is clearly a need for both teachers and students to develop critical digital and data literacies that enable them to engage as both critical consumers and producers of data, knowledge and practice" [23].

An additional step aligned with civic implications includes monitoring and studying evolving development of regulations surrounding the market and use of AI systems. E.g., EU law currently proposes that "AI systems used in education or vocational training, notably for determining access or assigning persons to educational and vocational training institutions or to evaluate persons on tests as part of or as a precondition for their education should be considered high-risk, since they may determine the educational and professional course of a person's life and therefore affect their ability to secure their livelihood. When improperly designed and used, such systems may violate the right to education and training as well as the right not to be discriminated against and perpetuate historical patterns of discrimination" [31].

We assert that we are on the cusp of even greater emergence of learning environments using AI technologies. These environments will evolve exponentially, along with our notions of agency. We encourage researchers to examine the tangling inherent throughout industry marketing artifacts, employing digital archives such as Fabric as a means to study and design AI learning environments with increased student and instructor agency.

\section{References}

[1] A.S. Brede, "Your science assistant", TEDxBinnenhof, https://www.youtube.com/watch?v=TzXpNEt5q$\underline{\mathrm{U} \& \mathrm{t}=53 \mathrm{~s}, 2016 .}$

[2] K. Crawford, Atlas of AI: Power, politics and the planetary costs of artificial intelligence, Yale University Press, 2021.

[3] N. K. Hayles, Unthought: The power of the cognitive nonconscious, The University of Chicago Press, 2017.

[4] S. Tosoni and T Pinch, Entanglements: Conversations on the Human Traces of Science, Technology, and Sound, MIT Press, 2016.

[5] L. Der and F. Fernandini, Archaeology of Entanglement, Routledge, 2016.

[6] G. Siemens, "The post-learning era in higher education: Human + machine", Educause Review, https://er.educause.edu/articles/2020/2/the-postlearning-era-in-higher-education-human-machine, 2020.

[7] B. Shneiderman, "Human-Centered Artificial Intelligence: Reliable, Safe \& Trustworthy”, International Journal of Human-Computer Interaction, 36(6), pp. 495-504, 2020.

[8] V. Dignum, Responsible Artificial Intelligence: How to Develop and Use AI in a Responsible Way, Springer Nature Switzerland, 2019.

[9] A. H. Duin and I. Pedersen, Writing Futures: Collaborative, Algorithmic, Autonomous.: Springer Nature Switzerland, 2021.

[10] E. Volini, B. Denny, and J. Schwartz, Superteams: Putting AI in the group, Deloitte Insights, https://www2.deloitte.com/uk/en/insights/focus/humancapital-trends/2020/human-ai-collaboration.html, 2020.

[11] J. Bryson, "Robots should be slaves", In: Y. Wilks (ed) Close engagements with artificial companions: key social, psychological, ethical and design issues, John Benjamins Publishing, Amsterdam, 2010.

[12] J. Danaher, "The philosophical case for robot friendship", Journal of Posthuman Studies, 3(1), pp. 5 24, 2019.

[13] D. Gunkel, "Other Things: AI, Robots, and Society", in Z. Papacharissi (ed.) A Networked Self and Human Augmentics, Artificial Intelligence, Sentience, Taylor and Francis, 2019.

[14] E.J. De Visser, R. Pak, and T.H. Shaw, "From 'automation' to 'autonomy': the importance of trust repair in human-machine interaction", Ergonomics, 61(10), pp. 1409-1427, 2018.

[15] Artificial Intelligence Index Report 2021, HumanCentered Artificial Intelligence, Stanford University, https://hai.stanford.edu/research/ai-index-2021. 
[16] K. Pelletier, M. Brown, D. C. Brooks, M. McCormack, J. Reeves, N. Arbino, A. Bozkurt, S. Crawford, L Czerniewicz, R. Gibson, K Linder, J. Mason, and V. Mondelli, 2021 EDUCAUSE Horizon Report: Teaching and Learning Edition, 2021.

[17] University of Technology in Eindhoven. "TU/e announces EAISI: new institute for intelligent machines", www.tue.nl,

https://www.tue.nl/en/our-

university/departments/electrical-

engineering/department/news/news-overview/11-072019-tue-announces-eaisi-new-institute-for-intelligentmachines/, 2019.

[18] National Science Foundation, "NSF partnerships expand National AI Research Institutes to 40 states", www.nsf.gov

https://www.nsf.gov/news/news_summ.jsp?cntn_id=30317 6, 2021.

[19] O. Zawacki-Richter, V.I. Marin, M. Bond, and F. Gouverneur, "Systematic review of research on artificial intelligence applications in higher education -where are the educators?", International Journal of Educational Technology in Higher Education, 16(39), 2019.

[20] G. Guan, J. Mou, and Z. Jiang, "Artificial intelligence innovation in education: A twenty-year data-drive historical analysis", International Journal of Innovation Studies, 4, pp. 134-147, 2020.

[21] A.M. Cox, "Exploring the impact of artificial intelligence and robots on higher education through literature-based design fictions", International Journal of Educational Technology in Higher Education, 18(3), https://doi.org/10.1186/s41239-020-00237-8, 2021.

[22] A.H. Duin and J. Tham, "The current state of analytics: Implications for learning management system (LMS) use in writing pedagogy", Computers and Composition, 55, 2020.

[23] N.B. Dohn, P. Jandric, T. Ryberg, and M. de Laat, (eds.). Mobility, data and learner agency in networked learning, Springer, 2020.

[24] D.R. Mullet, "A general critical discourse analysis framework for educational research", Journal of Advanced Academics, 29(2), pp. 116-142, 2018.

[25] P.W. Kim, "Ambient intelligence in a smart classroom for assessing students' engagement levels", Journal of Ambient Intelligence and Humanized Computing, 10, pp. 3847-3852, 2018.

[26] M. Murgia, "Emotion recognition: can AI detect human feelings from a face?", Financial Times, May 122021.

[27] L.F. Barrett, R. Adolphs, S. Marsella, A.M. Martinez, and S.D. Pollak, "Emotional expressions reconsidered: Challenges to inferring emotion from human facial movements", Psychological Science in the Public Interest, 20, pp. 1-68, 2019.

[28] Z. Papacharissi (ed.), A networked self and human augmentics, artificial intelligence, sentience, Routledge, 2019.

[29] M. Matulik, M. Vavrecka, and L. Vidovicova, "Edutainment software for the Pepper robot", ISCSIC 2020: Proceedings of the 2020 4th International
Symposium on Computer Science and Intelligent Control, ACM, 2020.

[30] T. Ryberg and C. Sinclair, "The relationships between policy, boundaries and research in networked learning", In T. Ryberg, C. Sinclair, S. Bayne, M. de Laat (eds.), Research, Boundaries, and Policy in Networked Learning, Springer, pp. 1-20, 2016.

[31] European Commission, Regulation of the European Parliament and of the Council laying down harmonised rules on artificial intelligence (Artificial Intelligence $A c t)$ and amending certain union legislative acts, https://eur-lex.europa.eu/legalcontent/ES/TXT/?uri=COM:2021:206:FIN, 2021.

\section{Appendix 1. Set of Video Advertisements}

1. Artifact title: Meet AI XPRIZE Semifinalist Iris.AI

Company: Iris.AI, 2020

Classification: Advertisement, Inventions, Mobile

Types of AI technologies: Artificial Intelligence (AI), Machine Learning, Virtual Assistants

2. Artifact title: OrCam Read Features Overview

Company: OrCAM, 2020

Classification: Advertisement, Inventions, Wearable

Types of AI technologies: Artificial Intelligence (AI), Computer Vision

3. Artifact title: WritingAssistant

Company: EnglishHelper, Inc., 2020

Classification: Advertisement, Inventions

Types of AI Technologies: Artificial Intelligence (AI), Machine Learning, Natural Language Processing (NLP), AI Writing Assistants, Virtual Assistants

4. Artifact title: Misty II Is Here

Company: MistyRobotics, 2019

Classification: Advertisement, Inventions, Robot

Types of AI Technologies: Robotics, Humanoid Robots, Social Robots, Personal Robots, Artificial Intelligence (AI), Deep Learning, Computer Vision

5. Artifact title: Introductory to Squirrel AI Learning Company: Squirrel AI, 2019

Classification: Advertisement, Inventions, mobile Type of AI Technologies: Artificial Intelligence (AI), AI Tutors, Machine Learning, Algorithms , Intelligent Adaptive Learning System (IALS)

6. Artifact title: AMBASSADOR by Waverly Labs Company: Waverly Labs, 2019

Classification: Advertisement, Inventions, Wearable Types of AI technologies: Artificial Intelligence (AI), Neural Networks

7. Artifact title: Little Sophia by Hanson Robotics

Company: Hanson Robotics, 2019

Classification: Advertisement, Inventions, Robot 
Types of AI technologies: Robotics, Humanoid Robots, Social Robots, Human-Robot Interaction (HRI), Artificial Intelligence (AI), Facial Recognition

8. Artifact title: Sophia the Robot's Journey: Reflections on 2018

Company: Hanson Robotics, 2018

Classification: Advertisement, Inventions, Robot

Types of AI technologies: Robotics, Humanoid Robots, Social Robots, Artificial Intelligence (AI), Human-Robot Interaction (HRI)

9. Artifact title: Xiaoyou robots made by Canbot

Company: Canbot, 2018

Classification: Advertisement, Inventions, Robot

Types of AI technologies: Robotics, Social Robots, Personal Robots, Humanoid Robots, HumanRobot Interaction (HRI), Artificial Intelligence (AI), Natural Language Processing (NLP)

10. Artifact title: Zenbo B2B Service Kicks Off | ASUS

Company: ASUS, 2018

Classification: Advertisement, Inventions, Robot

Types of AI technologies: Robotics, Humanoid Robots, Social Robots, Personal Robots, Artificial Intelligence (AI), Human-Robot Interaction (HRI), Facial Recognition

11. Artifact title: ProWritingAid: Your Personal Writing Coach

Company: Orpheus Technology Ltd, 2018

Classification: Advertisement, Inventions, mobile

Types of AI Technologies: Artificial Intelligence (AI), Machine Learning, Natural Language Processing (NLP), Virtual Assistants

12. Artifact title: Scholarcy - your digital research assistant

Company: Scholarcy, 2018
Classification: Advertisement, Inventions, mobile Types of AI Technologies: Artificial Intelligence (AI), Algorithms , Machine Learning, Virtual Assistants

13. Artifact title: Solution AI big data

Company: Find Solution AI, 2018

Classification: Advertisement, Inventions, mobile

Types of AI Technologies: Artificial Intelligence (AI), Facial Recognition, Emotion Detection

14. Artifact title: MondlyAR - learn languages in augmented reality

Company: Mondly Languages, 2018

Classification: Advertisement, Inventions, mobile

Types of AI Technologies: Artificial Intelligence (AI), Virtual Assistants, Speech Recognition,

15. Artifact title: emotix | Meet Miko — India's First Companion Robot

Company: Emotix Official, 2016

Classification: Advertisement, Inventions, Robot

Types of AI Technologies: Robots, Social Robots, Personal Robots, Robotics, Human-Robot Interaction (HRI), Artificial Intelligence (AI)

16. Artifact title: FOVE VR Headset: Tracks Subtle Eye Movements in Virtual Reality

Company: Fove, Inc., 2015

Classification: Advertisement, Inventions, wearable Types of AI Technologies: Artificial Intelligence (AI) 17. Artifact title: Jibo The World's First Family Robot Company: Jibo (no longer operating since 2019), 2014

Classification: Advertisement, Inventions, Robot

Types of AI Technologies: Artificial Intelligence (AI), Robotics, Humanoid Robots, Personal Robots, Social Robots, Natural Language Processing (NLP), Facial Recognition , Human-Robot Interaction (HRI) 